\title{
Tratamento da leishmaniose mucosa com sulfato de aminosidine: resultados de dois anos de acompanhamento
}

\author{
Mucosal leishmaniasis treatment with aminosidine \\ sulfate: results of two year \\ follow-up \\ Gustovo A.S. Romero, Hélio A. Lessa, Maria G.O. Orge, \\ Vanize O. Macêdo e Philip D. Marsden†
}

\begin{abstract}
Resumo Em 1996 foram avaliados clinicamente 20 dos 21 pacientes com leishmaniose mucosa, tratados em $1994 \mathrm{com}$ sulfato de aminosidine 16mg do sal/kg/dia, intramuscular, por 20 dias. Um paciente foi a óbito por causas não relacionadas com a leishmaniose mucosa. Dos 14 pacientes $(66,7 \% N=21)$ que inicialmente alcançaram a remissão completa dos sinais e sintomas durante os três primeiros meses de seguimento, sete $(50 \% \mathrm{~N}=14)$ permaneceram livres de doença por 24 meses e sete pacientes apresentaram recidiva neste período. 0 acompanhamento sorológico mostrou pobre correlação com a avaliação clínica.
\end{abstract}

Palavras-chaves: Leishmaniose mucosa. Sulfato de aminosidine. Leishmania (Viannia) braziliensis.

\begin{abstract}
In 1996, 20 of 21 patients with mucosal leishmaniasis, treated in 1994 with aminosidine sulfate, $16 \mathrm{mg} / \mathrm{kg} /$ day salt, by intramuscular injection for 20 days, were clinically evaluated. One patient died due to disease not related to mucosal leishmaniasis. Seven of 14 patients $(66,7 \% N=21)$ who achieved complete remission three months after treatment remained clinically cured 24 months later and seven relapsed in the same period $(50 \% N=14)$. Sorological follow-up showed poor correlation with the results of clinical examination.
\end{abstract}

Key-words: Mucosal leishmaniasis. Aminosidine sulfate. Leishmania (Viannia) braziliensis.

\footnotetext{
Núcleo de Medicina Tropical e Nutrição/Universidade de Brasília, Brasília, DF e Serviço de Imunologia do Hospital Universitário Prof. Edgar Santos/Universidade Federal da Bahia, Salvador, BA.

Órgão financiador: Fundo de Auxílio a Pesquisa do Distrito Federal (FAP-DF)

Endereço para correspondência: Dr. Gustavo A.S. Romero. Núcleo de Medicina Tropical e Nutrição/UnB. Caixa Postal 04-671, 70919-970 Brasília, DF.

Fax: (061) 273-2811.

e-mail: gromero @ guarany.cpd.unb.br

Recebido para publicação em 25/09/97.

† In memoriam.
} 
O sulfato de aminosidine é um aminoglicosídeo com atividade leishmanicida7 que tem sido utilizado nas diversas formas de leishmaniose, com eficácia variável2 13 14. Em 1994 foi realizado um estudo terapêutico aberto em 21 pacientes com leishmaniose mucosa (LM) ativa em área endêmica de Leishmania (Viannia) braziliensis $(\mathrm{LVb})^{11}$. Os resultados iniciais mostraram que $66,7 \%$ (14/21 pacientes) alcançaram a remissão completa dos sinais e sintomas da doença durante os três primeiros meses de acompanhamento. $\mathrm{A}$ avaliação trimestral durante o primeiro ano mostrou que quatro, dos 14 pacientes com boa resposta inicial, apresentaram recidiva. A taxa de cura clínica, definida como a ausência de sinais e sintomas da doença durante o primeiro ano de acompanhamento foi $48 \%$ (10/21 pacientes) ${ }^{10}$. Pelas evidências que sugerem que a infecção por $L V b$ pode persistir indefinidamente no hospedeiro humano3 612 e pela observação de episódios de recidiva da doença mucosa, anos após a cura clínica ${ }^{9}$, realizou-se a procura ativa dos 21 pacientes tratados com sulfato de aminosidine dois anos após o tratamento com o objetivo de detectar possíveis recidivas e completar a avaliação sorológica.

\section{MATERIAL E MÉTODOS}

Em 1996 foram avaliados 20 dos 21 pacientes com LM tratados em 1994 com sulfato de aminosidine, $16 \mathrm{mg}$ do sal $/ \mathrm{kg} /$ dia por 20 dias, intramuscular. Realizou-se a avaliação clínica para estabelecer a atividade da doença mucosa utilizando-se como critério de atividade a presença de edema, ulcerações e/ou infiltração granulosa ou vegetante da mucosa das vias aéreas superiores. Foi realizada a pesquisa de anticorpos anti-leishmania no soro com a técnica de imunofluorescência indireta (IFI) utilizandose como antígeno Leishmania (Leishmania) amazonensis MHOM/BR/86/BA1251. Durante os dois anos de acompanhamento todas as reações sorológicas foram realizadas com a mesma técnica pelo mesmo observador, diminuindo desta forma as potenciais variações na interpretação da prova.

Um paciente foi a óbito 3 meses antes de completar os dois anos de seguimento por problemas não relacionados com a doença mucosa. Este paciente encontrava-se sem doença ativa na avaliação realizada um ano após o tratamento com aminosidine.

Os pacientes que não apresentaram remissão completa da doença durante os três primeiros meses de acompanhamento ou que recidivaram durante o primeiro ano, receberam tratamento com antimonial pentavalente $(20 \mathrm{mg}$ de $\mathrm{SbV} / \mathrm{kg} / \mathrm{dia}$ por 30 dias). Uma paciente teve indicação de tratamento hospitalar com anfotericina B por falta de resposta adequada ao tratamento com antimonial.

Realizou-se a análise estatística dos títulos da IFI após a transformação logarítmica dos valores recíprocos e as comparações foram realizadas com a prova de $T$ de student pareada. Consideraram-se significativos títulos maiores que 1:16 e na transformação logarítmica, os negativos (a primeira diluição do soro avaliada foi $1: 16)$ receberam um valor de três para que análise não fosse prejudicada pela diminuição significativa de três diluições em pacientes que permaneceram com títulos negativos. Calculouse a concordância entre a atividade clínica da doença e os resultados da IFI utilizando o coeficiente Kappa. Utilizou-se a análise não paramêtrica de Kruskal Wallis para dois grupos (KWH) na comparação de médias quando as variâncias não foram homogêneas pela prova de Bartlett.

\section{RESULTADOS}

A Figura 1 mostra a evolução dos 14 pacientes que alcançaram a remissão completa no período de três meses após o tratamento e o índice acumulado de recidiva durante os 24 meses de acompanhamento. A taxa de cura clínica 24 meses após o tratamento com sulfato de aminosidine foi 33,3\% ( $N=21)$ e a taxa de recidiva $50 \%(\mathrm{~N}=14)$.

As médias geométricas (MGT) dos valores recíprocos da IFI, incluindo os pacientes que receberam tratamento com antimonial pentavalente ou anfotericina $B$, foram 64,0 ; 18,$9 ; 16,0 ; 28,0 ; 20,8$ e 17,5 zero, três, seis, nove, doze e vinte e quatro meses após tratamento. Houve diminuição significativa entre zero e três meses $(t=6,52 p<0,005)$. A Figura 2 mostra estes resultados em escala logarítmica com os respectivos intervalos de confiança 95\% (IC95\%).

As MGT calculadas excluindo os pacientes que receberam tratamentos alternativos foram 64,0; 18,$9 ; 15,1 ; 24,9 ; 24,2$ e 14,9 zero, três, seis, nove, doze e vinte e quatro meses após tratamento. Houve uma diminuição significativa entre zero e três meses ( $t=6,52 \mathrm{p}<0,005)$; aumento significativo entre seis e nove meses $t=-3,59$ $p<0,005)$ e diminuição significativa entre 12 e 24 


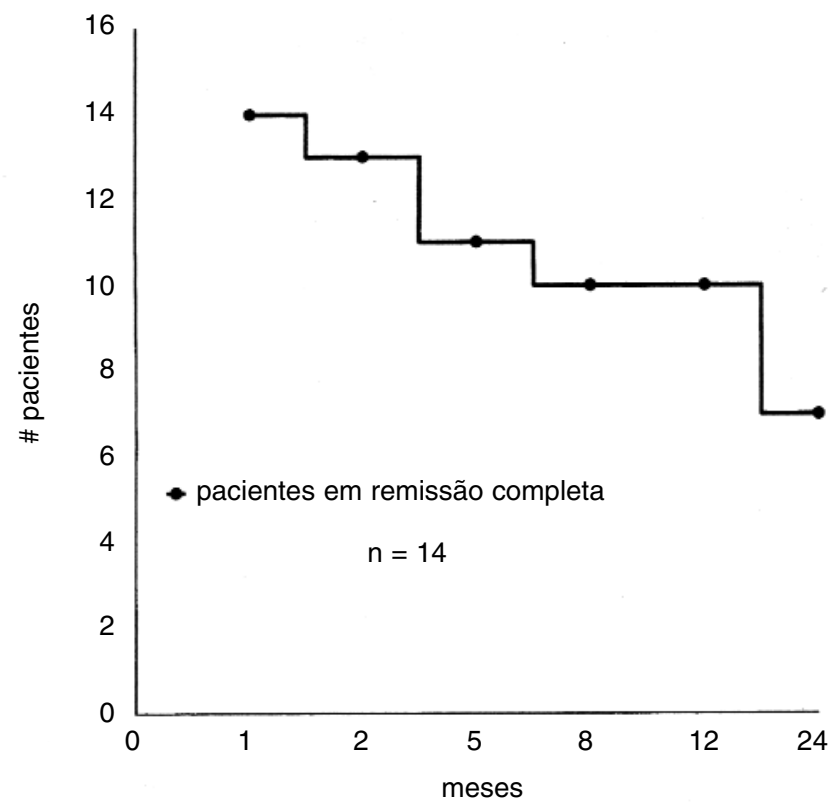

Figura 1 - Evolução dos 14 pacientes que alcançaram a remissão completa após tratamento com sulfato de aminosidine durante os 24 meses de acompanhamento.

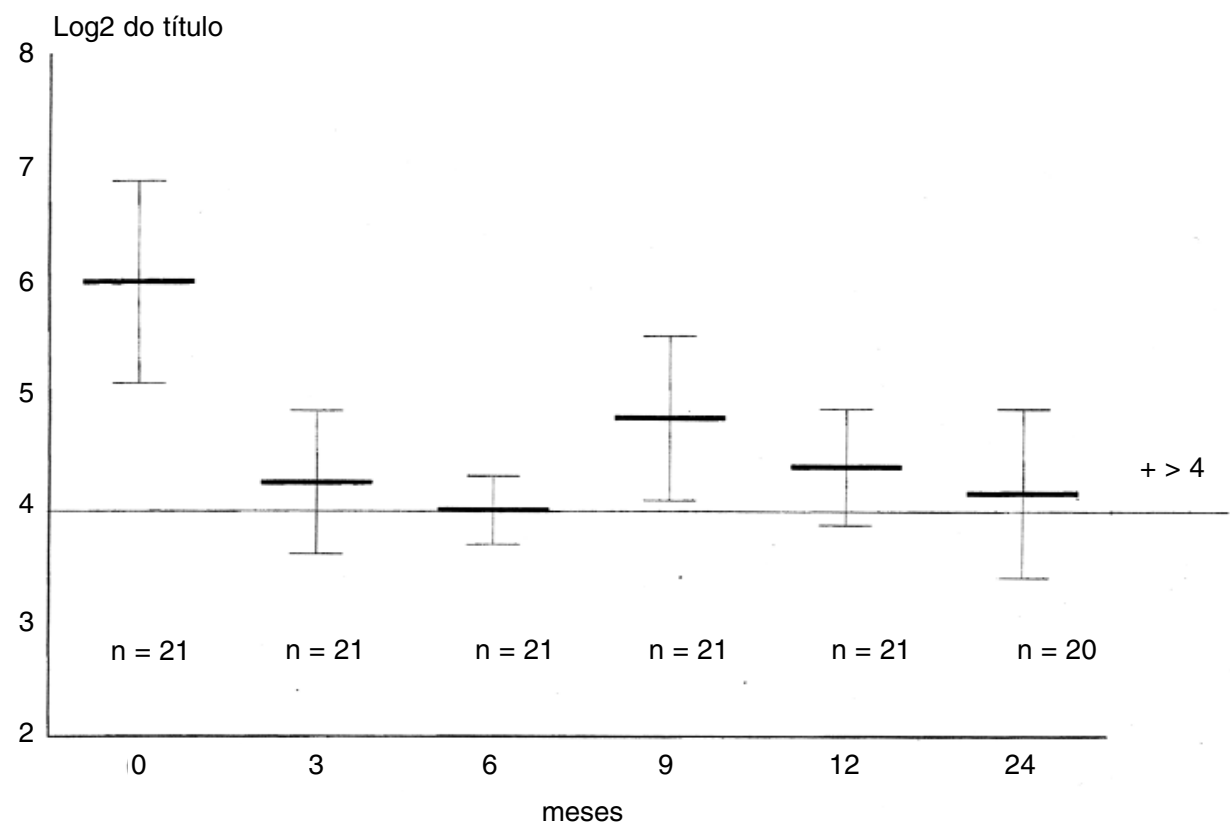

Figura 2 - Evolução sorológica dos 21 pacientes durante os 24 meses de acompanhamento. Média dos títulos da IFI (log2 do recíproco do título) com intervalo de confiança 95\% como valores máximo e mínimo. 
meses $(t=3,28 p<0,005)$. A Figura 3 mostra estes resultados com os respectivos intervalos de confiança 95\% (IC95\%).

A análise da concordância entre os títulos da IFI e a avaliação clínica mostrou que não existe uma boa correlação (Coeficiente Kappa = 0,25).
A Figura 4 mostra graficamente a distribuição dos valores dos títulos da IFI em relação com a atividade da doença.

Os títulos da IFI nas avaliações 12 e 24 meses após tratamento foram significativamente maiores em pacientes que apresentaram doença

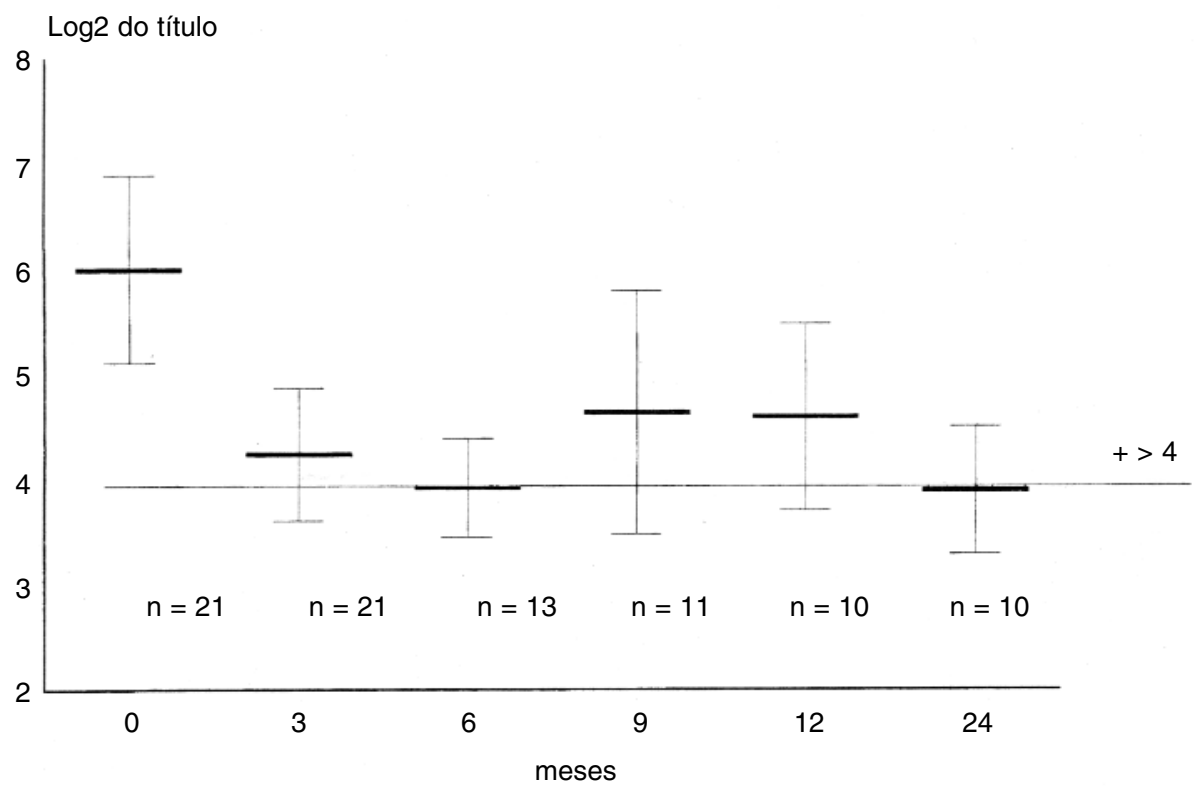

Figura 3 - Evolução sorológica dos 21 pacientes excluindo progressivamente os tratados com outras drogas por falha terapêutica ou recidiva. Média dos títulos da IFI (log2 do recíproco do título) e intervalo de confiança 95\% como valores máximo e mínimo.

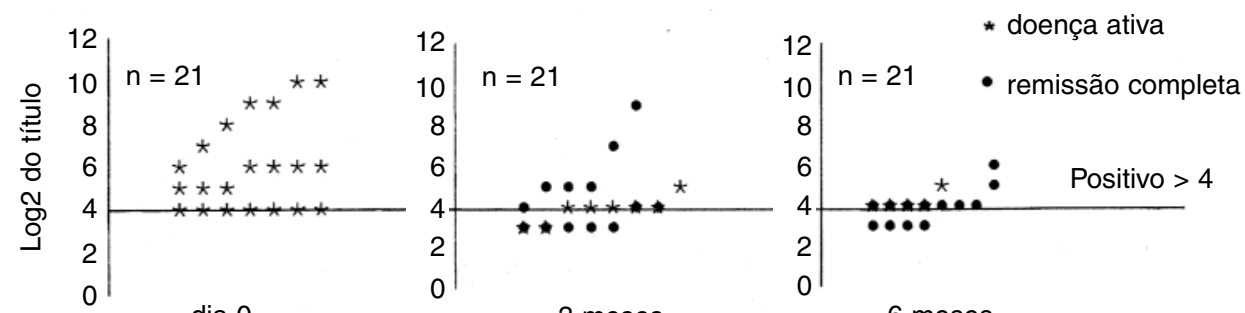

dia 0

3 meses

6 meses
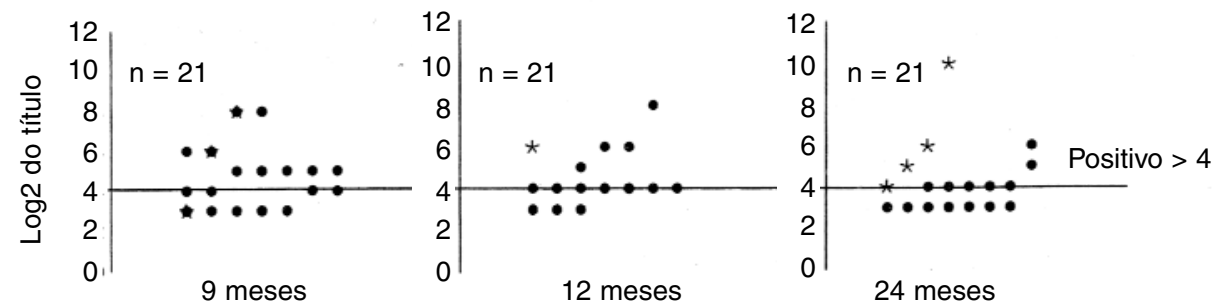

Figura 4 - Distribuição do Log2 dos valores recíprocos dos títulos da IFI correlacionados com a atividade da doença durante os dois anos de acompanhamento. 
ativa na avaliação de 24 meses $(\mathrm{KWH}=6,3 ; \mathrm{p}$ $=0,01$ aos 12 meses e $\mathrm{KWH}=6,6 ; p=0,01$ aos 24 meses) porém, o número de pacientes com doença ativa na avaliação final foi baixo (quatro pacientes) para estabelecer conclusões.

\section{DISCUSSÃO}

As características gerais dos pacientes estudados aparecem em detalhe na publicação dos resultados do primeiro ano de acompanhamento 10 . Os resultados obtidos durante os dois anos de seguimento mostraram que a eficácia do tratamento da LM com uma série de 20 dias de sulfato de aminosidine é baixa $(33,3 \%)$ e que está indicado o acompanhamento prolongado dos pacientes com este tipo de doença, pela possibilidade de recidiva. A observação qualitativa dos dados mostrou que seis dos sete pacientes que apresentaram recidiva não tinham sido tratados com drogas leishmanicidas antes do uso do sulfato de aminosidine e que os quatro pacientes com lesões múltiplas que atingiram nariz, faringe e laringe apresentaram também este tipo de evolução. Estas observações podem sugerir que existe a possibilidade de efeito cumulativo das drogas leishmanicidas e que o período em que o efeito poderia ser observado é maior que os três meses estabelecidos nos critérios de inclusão deste trabalho para eliminar a interferência de outras drogas na ação terapêutica do sulfato de aminosidine. Os pacientes que não recidivaram poderiam ter uma carga parasitária menor por efeito residual de outros tratamentos. O acometimento múltiplo, pela sua gravidade, também poderia apresentar uma resposta menos satisfatória.

Os resultados da IFI mostraram pobre correlação com a atividade da doença, achado que limitou a interpretação dos resultados durante o seguimento. Embora o estudo não fosse especialmente planejado para obter conclusões sobre o comportamento sorológico, os resultados sugerem que a interpretação da sorologia deve ser cautelosa nestes pacientes.

Os estudos terapêuticos da LM com antimonial pentavalente têm mostrado resultados variáveis e até hoje não permitem saber com certeza qual é a eficácia da droga neste tipo de apresentação clínica4 5 . O pequeno número de casos de LM observado dificulta a realização de estudos comparativos que permitam obter conclusões sobre a eficácia das drogas alternativas. Consideramos que um estudo multicêntrico poderia resolver as incógnitas que prevalecem até hoje. O uso de mais de uma série de tratamento com aminosidine possui uma limitação importante pela toxicidade renal e auditiva. O uso combinado ou sequencial desta droga junto ao antimonial pentavalente constitui a próxima abordagem no estudo deste medicamento na doença mucosa.

Sugerimos que o sulfato de aminosidine seja utilizado como alternativa terapêutica quando exista falta de resposta ou contra-indicação para o uso de antimonial pentavalente

\section{AGRADECIMENTO}

Ao Sr. Ednaldo Lago da Fundação Nacional de Saúde, Ministério da Saúde, pela ajuda na procura ativa dos pacientes.

\section{REFERÊNCIAS BIBLIOGRÁFICAS}

1. Badaró R, Reed SG, Carvalho EM. Immunofluorescent antibody test in American visceral leishmaniasis: sensitivity and specificity of different morphological forms of two Leishmania species. The American Journal of Tropical Medicine and Hygiene 32:480484, 1983.

2. Correia D, Macêdo VO, Carvalho EM, Barral A, Magalhães AV, Abreu MVA, Orge MGO, Marsden PD. Estudo comparativo entre antimoniato de meglumina, isotionato de pentamidina e sulfato de aminosidine, no tratamento de lesões cutâneas primárias causadas por Leishmania (Viannia) braziliensis (L(V)b). Revista da Sociedade Brasileira de Medicina Tropical 29:447453, 1996.

3. Delgado O, Guevara P, Silva S, Belfort E, Ramírez JL. Follow-up of a human accidental infection by Leishmania (Viannia) braziliensis using conventional immunologic techniques and polymerase chain reaction. The American Journal of Tropical Medicine and Hygiene 55:267-272, 1996.

4. Franke ED, Llanos-Cuentas A, Echevarria JE, Cruz MF, Campos P, Tovar AA, Lucas CM, Berman JD. Efficacy of 28-day and 40-day regimens of sodium stibogluconate (Pentostam) in the treatment of mucosal leishmaniasis. The American Journal of Tropical Medicine and Hygiene 51:77-82, 1994.

5. Franke ED, Wignall FS, Cruz ME, Rosales E, Tovar AA, Lucas CM, Llanos-Cuentas A, Berman JD. Efficacy and toxicity of sodium stibogluconate for mucosal leishmaniasis. Annals of Internal Medicine 113:934-940, 1990.

6. Guevara P, Ramírez JL, Rojas E, Scorza JV, González N, Añez N. Leishmania braziliensis in blood 
30 years after cure. The Lancet 341:1341, 1993.

7. Neal RA. The effect of antibiotics of the neomycin group on experimental cutaneous leishmaniasis. Annals of Tropical Medicine and Parasitology 62:5462, 1968.

8. Netto EM, Marsden PD, Llanos-Cuentas EA, Costa JML, Cuba CC, Barreto AC, Badaró R, Johnson WD, Jones TC. Long-term follow-up of patiens with Leishmania (Viannia) braziliensis infection and treated with Glucantime. Transactions of the Royal Society of Tropical Medicine and Hygiene 84:367370, 1990.

9. Oliveira MRF, Macêdo VO, Carvalho EM, Barral A, Marotti JG, Bittencourt A, Abreu MVA, Orge MGO, Lessa HÁ, Marsden PD. Estudo evolutivo da leishmaniose mucosa (7-17 anos de seguimento) causada por Leishmania (Viannia) braziliensis em Três Braços, Bahia. Revista da Sociedade Brasileira de Medicina Tropical 28:325-332, 1995.

10. Romero GAS, Lessa HÁ, Macêdo VO, Carvalho EM, Barral A, Magalhães AV, Orge MGO, Abreu MVA, Marsden PD. Estudo terapêutico aberto com sulfato de aminosidine na leishmaniose mucosa causada por Leishmania (Viannia) braziliensis. Revista da Sociedade Brasileira de Medicina Tropical 29:557565, 1996.

11. Rosa AC, Cuba CC, Vexenat A, Barreto AC, Marsden PD. Predominance of Leishmania braziliensis in the regions of Três Braços and Corte de Pedra, Bahia, Brazil. Transactions of the Royal Society of Tropical Medicine and Hygiene 82:409-410, 1988.

12. Saravia NG, Weigle K, Segura I, Giannini SH, Pacheco R, Labrada LA, Gonçalves A. Recurrent lesions in Leishmania braziliensis infectionreactivation or reinfection? The Lancet 336:398-402, 1990.

13. Soto J, Grogl M, Berman J, Olliaro P. Limited efficacy of injectable aminosidine as single-agent therapy for Colombian cutaneous leishmaniasis. Transactions of the Royal Society of Tropical Medicine and Hygiene 88:695-698, 1994.

14. Thakur CP, Bhowmick S, Dolfi L, Olliaro P. Aminosidine plus sodium stibogluconate for the treatment of Indian kala-azar: a randomized dosefinding clinical trial. Transactions of the Royal Society of Tropical Medicine and Hygiene 89:219-223, 1995. 